\title{
Predicting the Conformational Variability of Abl Tyrosine Kinase using Molecular Dynamics Simulations and Markov State Models
}

\author{
Yilin Meng ${ }^{1}$, Cen Gao², David Clawson², Shane Atwell ${ }^{3}$, Marijane Russell ${ }^{4}$, Michal Vieth ${ }^{4}$, \\ and Benoît Roux ${ }^{1, *}$ \\ ${ }^{1}$ Department of Biochemistry and Molecular Biology, University of Chicago, Chicago, IL, 60637, \\ USA \\ 2Discovery Chemistry Research and Technologies, Eli Lilly and Company, Lilly Corporate Center, \\ Indianapolis, IN, 46285, USA \\ ${ }^{3}$ Applied Molecular Evolution, Eli Lilly and Company, Lilly Biotechnology Center, 10290 Campus \\ Point Drive, San Diego, CA, 92121, USA \\ ${ }^{4}$ Discovery Chemistry Research and Technologies, Eli Lilly and Company, Lilly Biotechnology \\ Center, 10290 Campus Point Drive, San Diego, CA, 92121, USA
}

\section{Abstract}

\begin{abstract}
Understanding protein conformational variability remains a challenge in drug discovery. The issue arises in protein kinases, whose multiple conformational states can affect the binding of smallmolecule inhibitors. To overcome this challenge, we propose a comprehensive computational framework based on Markov State Models (MSMs). Our framework integrates the information from explicit-solvent molecular dynamics simulations to accurately rank-order the accessible conformational variants of a target protein. We tested the methodology using Abl kinase with a reference and blind-test set. Only half of the Abl conformational variants discovered by our approach are present in the disclosed X-ray structures. The approach successfully identified a
\end{abstract}

\footnotetext{
*Corresponding author: roux@ uchicago.edu, Gordon Center for Integrative Science, 929 E $57^{\text {th }}$ Street, Room W323B, Chicago, IL, 60637, USA, Telephone: 1-773-834-3557, Fax: 1-773-702-0439.

Author Contributions

Y.M., M.V., and B.R. conceived and designed research. M.V. and B.R. supervised the research. Y.M. performed simulations and constructed the MSMs. D.C. refined and analyzed the structure. S.A. designed the construct. M.R. created the construct. C.G. and M.V. analyzed the Lilly internal data. All authors discussed results and wrote the manuscript.

Supporting Information

Description of experimental details was given in the supporting information. Table S1 lists the collective variables that were utilized to featurize the conformational variation in the kinase domain. A brief summary of the final MSMs is given in Table S2. Table S3 lists the statistics of the final model of mAb11/WHI-P154 complex. Figure S1 illustrates the distribution of the percentage of sequence identity relative to wild-type human c-Abl tyrosine kinase. Figure S2 shows the conformational sampling of the phase space before the string method calculations were employed. Figure S3 illustrates the comparison of the conformational sampling of the phase space in the reference and the blind-test simulations. Figure S4 summarizes results of model selection based on the Markovianity of each pair of $K$ value and the initial assignment of the centroids. Figure S5 gives a cartoon illustration of the representative structure of the reference coordinates used to define the structural ensemble associated with all 16 macrostates superimposed. Figure S6 illustrates the linear fitting of the free energy values from the blind-test (X-ray based) set with respect to those from the reference (homology based) set. Figure S7 illustrates the closest macrostates based on variable regional all-atoms RMSD. Figure S8 shows the probability density of the Ca-atoms RMSD in the variable region with respect to macrostate 16, and the corresponding 1D-PMF. Figure S9 illustrates the correlation of variable region RMSD with the binding site Tanimoto shape distance. Results from the cluster analysis of the P-loop conformations in c-Abl X-ray structures were summarized in Figure S10. This information is available free of charge via the Internet at http://pubs.acs.org.
} 
protein conformational state not previously observed in public structures, but evident in a retrospective analysis of Lilly in-house structures: the X-ray structure of Abl with WHI-P154. Using MSM-derived model, the free energy landscape and kinetic profile of Abl was analyzed in detail highlighting opportunities for targeting the unique metastable states.

\section{Graphical Abstract}

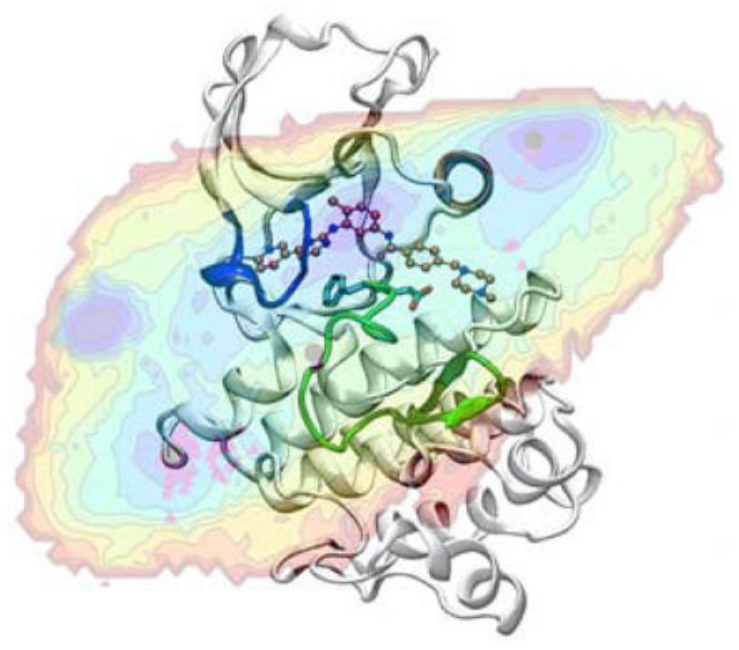

\section{INTRODUCTION}

Structure-based drug design (SBDD) encompasses experimental and computational methodologies aimed at discovering and refining small-molecule inhibitors of specific therapeutic targets by relying on the information from atomic complex structures and molecular interactions. ${ }^{1-2}$ These methodologies have the potential to vastly accelerate and drive drug discovery. Crystallographic information combined with virtual screening of existing and designed ligands can deliver small molecule starting points and binding poses in all phases of a drug discovery project. ${ }^{1-6}$ Nonetheless, it is apparent that, among other things, accounting for protein flexibility and accurate free energy determinations must be overcome to make SBDD a reliable tool for the pharmaceutical industry. Protein kinases are excellent prototypes for investigating new computational strategies. They are highly validated drug targets and exist in multiple conformational states with a vast amount of available structural data. The human genome encodes more than 500 protein kinases, making the kinome one of the largest gene families. ${ }^{7}$ Protein kinases, when in the active conformational state, catalyze the transfer of the $\gamma$-phosphate of an adenosine triphosphate (ATP) molecule to substrate protein. The phosphorylation of a substrate protein can result in a functional change, modification of cellular localization and play an important role in cellular signaling. Phospho-signalling is linked with a broad range of diseases, especially cancers. For this reason, they are major therapeutic targets and the discovery of kinasespecific inhibitors is intensely pursued by the pharmaceutical industry.

One of the critical challenges for successful SBDD efforts stems from the inherent flexibility of protein targets. Proteins exist in multiple conformational states and only some of them are 
observed by X-ray crystallography. Freezing the protein in unique states might lead to more desirable pharmacology for small molecules. When a crystal structure of a protein-ligand complex is available, researchers will often use a variety of well-established modeling tools to support lead generation and optimization efforts. One of the most common applications of SBDD is to use the inferred or crystallographic ligand-binding mode to design analogs and devise chemistry strategy. If no crystal structure is available, homology models can be used as the basis for future modeling, docking, and optimization. These conventional methods can perform fairly well if the binding pocket of the target protein has a single dominant conformation. ${ }^{1-2,5}$ However, conventional docking and scoring often fall short when a protein pocket is flexible and can adopt multiple distinct conformations. Experimental studies have demonstrated that protein kinases can adopt multiple conformational states, and that such structural variability is essential to their function and regulation. Furthermore, the relative propensity of these conformational variants affects the binding affinity and modality of inhibitors. Exploiting the structural variability in designing inhibitors targeting protein kinases has become a discovery strategy. Pursuing allosteric inhibitors, which bind to target kinases outside of the ATP-binding pocket led to multiple compounds in advanced clinical development targeting MEK, MAPK1 and AKT kinases. ${ }^{8}$ Kinase structural variability is partly reflected in the diversity of structures in the protein data bank (PDB). So far, over 200 out of the over 500 known protein kinases in the human kinome have been crystallized in at least one conformational state. Still, many important protein kinase targets are either unavailable or under-represented in the PDB. Even when multiple X-ray structures are available, our knowledge of all the relevant conformations remains limited, thus hindering SBDD. A computational methodology that is able to reliably generate (and predict the ranking of) the missing structures will greatly enhance the applicability of standard SBDD tools and enable new chemistry strategies.

A powerful framework to treat structural variability of complex biomolecular systems is provided by Markov state models (MSMs). ${ }^{9-12}$ The idea is to exploit the information from a large number of intermediate-length all-atom explicit-solvent molecular dynamics (MD) trajectories to construct a reduced stochastic model of the system (see Bowman et al. ${ }^{12}$ for a detailed review). This framework has been used to describe a variety of molecular processes. ${ }^{13-21}$ A recent study of the conformational plasticity within the apo-Bruton's tyrosine kinase (BTK) can exemplify the application of MD and MSM in biological macromolecules: Sultan et al. investigated conformational variants that are conserved kinome-wide. ${ }^{20}$ MSMs were constructed to connect conformational states and to characterize the thermodynamic and dynamic properties. Briefly, an MSM consists of a set of conformational microstates and a matrix of transition probabilities (or, equivalently, transition rates) between them (i.e., a master equation), which can be used to fully describe the system at long timescales. An eigenvalue-eigenvector decomposition of the transition matrix yields the equilibrium probability distribution of the microstates (i.e. thermodynamic information of the target protein) and timescales of the transitions (i.e. kinetic information). MSMs allow the mapping of stable conformations and automatic discovery of metastable states ${ }^{22-24}$ thus providing an excellent framework to explore an unknown conformational space through an adaptive sampling strategy. ${ }^{25}$ Furthermore, a number of advances based on the string method $^{26-27}$ and enhanced sampling simulations ${ }^{28-30}$ can be leveraged to create a complete 
MSM-based computational methodology that is able to overcome conformational challenges within the SBDD projects.

The conformational variability of the c-Abl/Abl1, illustrated in Figure 1, was evaluated to showcase the capability of our approach to predict and rank-order its multiple conformations based on their relative stability (i.e. a potential of mean force with respect to the index of the conformational state). For the sake of simplicity, we focused on the c-Abl kinase domain (KD) and tried to generate all accessible conformations with their thermodynamic rankorder without using any c-Abl structures. We then compared these results with those obtained from simulations that relied on known X-ray structures of c-Abl. The example of conformational variability observed for c-Abl and calculated with our method provides a good example of the situation facing many SBDD projects and advances in computational treatment described here are likely to have a broad impact.

\section{METHODS AND COMPUTATIONAL DETAILS}

\section{Protein Structure Preparation}

In theory, the set of initial conformations to seed the MSM can be assembled from five different sources. First, available X-ray structures of the protein target in the PDB are included as part of the set of seed conformations. Second, available X-ray structures of relevant homologs from the PDB are used to generate homology models. Third, we generate previously unobserved combination of conformational variants via "piecewise-mixing" of conformational variants (pmcv) taken from different X-ray structures in order to further complement the initial set from the PDB. For example, the P-loop in the c-Abl-Gleevec complex structures (PDB 1IEP) is in a kinked conformation. Even assuming that the other motifs (DFG, A-loop, Spines, etc) were to keep their conformation, we suspect that the Ploop could also be in an extended conformation. This plausible state is not observed in the PDB. So, we implant the extended conformation of the P-loop into in the 1IEP structure, a kind of "Frankenstein-like" conformation that combines known elements taken from different kinases. In practice, this was carried out via MODELLER ${ }^{31}$ and targeted molecular dynamics (TMD) simulations. ${ }^{32}$ The generation of alternative structures can also be enhanced by modeling methods such as Rosetta. ${ }^{33}$ Fourth, the string method is used to connect remote states separated by large free energy barriers, which lack intermediate conformations to seed the MD simulations. Fifth, as the work on the kinase family proceeds, we will also harvest relevant macrostates from previous MSMs as a bootstrap procedure to further enhance the set of initial conformations for a new uncharacterized kinase. For example, the microstates discovered in an MSM of Abl can be used as seeds to generate the aggregated conformational sampling of an MSM for Syk or other kinases. More generally, in an iterative bootstrapping procedure, the information from the MSM constructed for any member of the kinase family may be exploited to provide a "basis set" of putative initial microstates to seed the MD simulations for constructing the MSM for a new uncharacterized kinase.

The key question is whether the above "multi-source seeding strategy" for constructing MSM can successfully discover all the relevant conformational variants for an uncharacterized kinase. We put this question explicitly to the test by comparing an MSM of 
c-Abl (the apo-form) constructed from native X-ray structures of c-Abl (source \#1), with a second MSM constructed without any prior knowledge about c-Abl. We used models generated by homology (source \#2) and by pmcv (source \#3) for the second MSM.

Essentially, the second MSM is a blind test that does not include any information from X-ray structures of c-Abl, while the first MSM serves as a reference to be compared with. The string method calculations have been applied to both the reference set and the blind-test set (i.e. source \#4 is applicable to both sets). The string method calculations were aimed to improve the sampling of the conformational space, and hence the quality of the MSMs, for both sets.

The Abelson non-receptor tyrosine kinase (Abl1, or c-Abl), expressed by the ABL1 gene in human, contains a DNA-binding region (residue 869 to 968) and an F-actin-binding region (residue 953-1130), in addition to the tyrosine kinase core region (residue 61 to 531), which is flanked by an N-terminal "cap" region ( $\mathrm{N}$-cap, residue 2 to 60). The tyrosine kinase core of Abl1 displays a similar architectural scaffold to the Src family tyrosine kinases (SFKs). This region also contains a Src-homology 3 (SH3) and a Src-homology 2 (SH2) regulatory domain, followed by a catalytic domain (kinase domain, KD). Furthermore, a linker region is present that connects the $\mathrm{SH} 2$ and the KD. The KD is the focus of this study. A more detailed description of the structure and regulation of c-Abl can be found at Panjarian et al. ${ }^{34}$ The protein KD structures from the protein data bank (PDB, accessed August 2015) were used to create the reference and the blind-test sets. Among them, 46 and 3175 (80 and 5527 individual protein structures after splitting chains, if a PDB id has multiple ones) belong to the reference and the blind-test set, respectively. The homology modeling of c-Abl structures was carried out with MODELLER version 9.16. ${ }^{31}$ A pairwise sequence alignment relative to c-Abl was performed for the KD structures in the blind-test set. The histogram of the percentage of sequence identity is given in Figure S1. To ensure the quality of the structural models (the predicted c-Abl structures) yielded from homology modeling, a lower bound of $30 \%$ sequence identity was chosen (i.e. the protein $\mathrm{KD}$ whose percentage of sequence identity is above $30 \%$ relative to c-Abl was utilized as source \#2). A truncation of the percentage of sequence identity relative to c-Abl to $30 \%$ reduced the number of structural templates to 1128 protein KDs. One should note that many structural templates contain missing residues in the KD, especially in the activation loop (A-loop) region. These gaps were filled with MODELLER. ${ }^{35}$ Visual inspection of the c-Abl structural models was performed to remove unrealistic models. To further analyze the conformational variability of the c-Abl structures in both sets and to reduce the number of initial structures to seed the MD simulations, cluster analysis utilizing a hierarchical algorithm with the Ward linkage was performed. 10 and 150 clusters were eventually selected for the reference and the blindtest set respectively, and the structures of the centroids served as the starting structures for the first batch of the MD simulations.

\section{Simulation Details}

All MD simulations of c-Abl were performed using Amber14 with GPU acceleration. ${ }^{36-37}$ The apo-form of the isolated KD in the absence of the regulatory SH3-SH2-linker tandem (i.e., residues W235 to T498, c-Abl splice 1a numbering) was considered in the present simulations with unphosphorylated Y397 in the A-loop. Amber ff14SB force field was 
utilized for the protein. ${ }^{38}$ The all-atom system was solvated in a truncated octahedral simulation cell of TIP3P water molecules. ${ }^{39}$ The size of the simulation cell was chosen to extend at least $8 \AA$ away from the surface of the protein. Eight $\mathrm{Na}^{+}$ions were inserted into the system to neutralize the total charge. The final system contains approximately 33,000 atoms. Prior to any production run, each system was energy minimized for 2000 steps using the steepest descent algorithm, equilibrated in a canonical (NVT) ensemble for 400 ps with a positional restraint on each protein heavy atom (the force constant is $5 \mathrm{kcal} / \mathrm{mol} \cdot \AA^{2}$ ), and further equilibrated in an isothermal-isobaric (NPT) ensemble for $10 \mathrm{~ns}$ with a positional restraint on each $\mathrm{Ca}$ atom (the force constant is $0.5 \mathrm{kcal} / \mathrm{mol} \cdot \AA^{2}$ ). Each MD simulation in the production stage was performed in the NPT ensemble and was propagated for $100 \mathrm{~ns}$ without any form of restraint. The pressure and temperature were controlled by Monte-Carlo barostat and Berendsen thermostat and kept at 1 atmosphere pressure and $300 \mathrm{~K}$, respectively. ${ }^{40}$ Periodic boundary conditions (PBC) were applied to all MD simulations and the Particle Mesh Ewald (PME) was used to treat long-range non-bonded interactions. ${ }^{41}$ The short-range non-bonded interactions were truncated at $8 \AA$, with no switching function employed. Covalent bonds involving hydrogen atoms were constrained at their equilibrium distances with the SHAKE algorithm, ${ }^{42}$ and a 2 fs time step was used throughout all simulations.

Although the MD simulations in the production stage started with only 10 and 150 structures for the reference and the blind-test set respectively, 30 and 5 different initializations of the velocity at $300 \mathrm{~K}$ were assigned to each structure in the reference and the blind-test set. Therefore, 300 and 750 individual MD trajectories were accumulated for the reference and the blind-test set after the first batch of simulations. Inspection of the sampling of the conformational space suggested that the string method calculations ${ }^{27}$ were necessary to accelerate the sampling and to construct MSMs effectively (also observed in the investigation of c-Src activation). ${ }^{43}$ Otherwise, MD propagations can be confined to several stable but isolated energy basins (see Figure S2), which results in discarding data (essentially repeating much of the conformational sampling) when constructing MSMs. Furthermore, an adaptive sampling strategy developed by the Bowman group (the FAST algorithm) was employed to facilitate connecting isolated regions and to improve the statistical accuracy of conformational sampling. ${ }^{44}$ Eventually, an aggregated MD sampling of $\sim 400 \mu$ s was generated from each set. The projections of MD simulations in the final sets onto the top 2 time-lagged independent component (tICs) are displayed in Figure S3. As shown in the figure, not only is the conformational space connected, the overall shapes for two different sets resemble each other.

\section{Construction of MSMs and model selection}

Data analysis and MSM construction were performed with MSMBuilder version 3.6. ${ }^{45}$ Molecular descriptors/features are extracted to coarse-grain the conformational variability for the dataset. This process is described as "featurization". The coarse-grained feature space has a much lower dimensionality and is still able to retain the important dynamics when compared to the original conformational space. One advantage of utilizing a lowdimensional feature space is that the impact of "curse of dimensionality" can be reduced. An MSM is then constructed based on the feature space. In our case, the feature space contains 
distances characterizing the conformations of the aC-helix, DFG-motif, P-loop, A-loop, and the regulatory spine (R-spine). All the distances utilized as the features are listed in Table S1. The A-loop is a long ( 20 amino acid) and flexible region in c-Abl and it is not intuitive how to select a small subset of the distances to further coarse-graining the A-loop motion. Thus the time-lagged independent component analysis (tICA) technique with kinetic mapping was applied to the A-loop in order to further reduce the dimensionality of the feature space. ${ }^{46-48}$ tICA was developed to extract slow collective motions in a molecule based on the de-autocorrelation time of each collective motion. ${ }^{47}$ The lag-time utilized in our tICA decomposition was $1 \mathrm{~ns}$. Eventually, seven distances (characterizing the dynamics of the P-loop, aC-helix, DFG-motif, and R-spine) and five tICs (characterizing the motion of A-loop) have been selected to form the final feature space. The conformational microstates in an MSM were then generated by partitioning the featurized MD data with the mini-batch $K$-Means clustering algorithm. ${ }^{49}$

Two hyperparameters requiring special attention are the number of clusters ( $K$, as the name of the algorithm indicated) and the random initial assignment of the cluster centroids. A series of $K$ values ( 9 in total) was scanned and five different random initial assignments of the centroids were utilized for each individual $K$ value. To select the "best" MSMs, we monitored the Markovianity of each pair of $K$ value and the initial assignment of the centroids and the implied timescales yielded from the corresponding MSM. The "best" MSMs should be Markovian (i.e. the implied timescales are stable regardless of the lagtime) and the eigenvalues of the transition matrix should be maximized in accordance with the variational formulation of kinetics introduced by Noé and Nüske (i.e. the implied timescales are the longest among the MSMs). ${ }^{50-51}$ The results of the longest implied timescale from each pair are given in Figure S4. Eventually, five MSMs that meet our criteria were selected (details in Table S2) for further analysis.

\section{Computation of the thermodynamic ranking of key conformations}

16 key macrostates defined from conformational variants of the aC-helix (in, out), the DFGmotif (in, out), the P-loop (kinked, extended), and the A-loop ( $a, \beta$, extended) were used for these MSMs. To compute the free energy of each macrostate, the MSM microstates were lumped into the macrostates based on the Voronoi tessellation of the feature space, i.e. each MSM microstate was partitioned to its nearest macrostate in the feature space. Once the partition of the MSM microstates to macrostates was finished, the corresponding equilibrium probabilities were added and utilized as the equilibrium probability of that macrostate.

\section{Assignment of Abl X-ray structures to macrostates}

The Abl X-ray structures in the public domain as well as in the Lilly internal structural database were assigned to the macrostates. Each one of the Abl X-ray structures with at least 38 residues of the 45 residues in the variable region was superimposed with conserved core algorithm ${ }^{52}$ and all-heavy-atom RMSD was computed for variable regions. Only the Abl structures with resolution below $2.6 \AA$ were considered, thus resulting in 388 ones in the final set. The all-heavy-atom RMSD values ranged from 0.046 to $15.75 \AA$. The algorithm uses backbone conserved core (within $1 \AA$ RMSD) as superposition region. The macrostate 
corresponding to the smallest all-atom RMSD value was assigned to each X-ray structure.

The all-atom RMSD values for closest macrostate ranged from 0.046 to $6.1 \AA$.

\section{Binding site shape similarity}

The shape similarity of the different binding sites was calculated using a grid-based approach. Specifically, the grid is generated with a mesh spacing of $0.5 \AA$ in the active site, defined as $7 \AA$ around the ligand in a reference Abl X-ray structure (imatinib-bound, PDB id: $2 \mathrm{HYY}^{53}$ ). The structures of the $16 \mathrm{MSM}$ macrostates were superimposed to the reference structure by aligning on the active site residues. For each macrostate, the binding pocket is characterized by the largest continuous set of grid points that do not clash with protein atoms. Electrostatic and the van der Waals (vdW) potential of each grid point was computed in Molecular Operation Environment (MOE) version 2016 and converted to binary grid occupancy for each structure ${ }^{54}$. The similarity $S$ of two binding sites $A$ and $B$, is defined as the Tanimoto similarity between two set of grid points,

$$
S(A, B)=\frac{\sum_{i=1}^{N \text { grid }} X_{i A} X_{i B}}{\sum_{i=1}^{N \text { grid }} X_{i A}^{2}+\sum_{i=1}^{N \text { grid }} X_{i B}^{2}-\sum_{i=1}^{N g r i d} X_{i A} X_{i B}}
$$

where $X_{i A}$ indicates grid occupancy by active site of structure A ( 0 or 1$)$. According to this metric, two identical binding sites will have a $S$ of 1.0 whereas two very dissimilar sites will have $S$ of zero. In practice, the points are redistributed to a $0.25 \AA$ common grid using trilinear interpolation for the matching.

\section{RESULTS AND DISCUSSION}

\section{Comparison of the thermodynamic rankings from the reference set and the blind-test set}

In the current study, a multi-source seeding strategy was utilized to generate starting structures ("seeds") for simulation. To test whether the computational framework could discover all the relevant conformational variants, we created two separate data sets as seed structures for simulation. The "reference" set was constructed by using publicly available $\mathrm{Abl} \mathrm{X}$-ray structures as seeds and the "blind-test" set only used models based on non-Abl PDB structures. 4040 and 4118 trajectories of $100 \mathrm{~ns}$ were accumulated for the reference and the blind-test sets respectively, yielding $\sim 400 \mu$ s of trajectories for each set. The results of the simulations are summarized in Figure 2a. We calculated and compared the free energies of 16 key macrostates defined from conformational variants of the aC-helix (in, out), the DFG-motif (in, out), the P-loop (kinked, extended), and the A-loop (a, $\beta$, extended) utilizing our MSM framework. Each of these 16 macrostate corresponds to a structural ensemble constructed from a Voronoi tessellation, each cell being defined by a representative reference coordinate set of the protein, and the free energy of the 16 macrostates is determined by accumulating the total equilibrium probabilities, $\pi_{i}$, over all the microstates ascribed to the Voronoi cell of a given macrostate. A pictorial representation of all 16 macrostates is given in Figure 2b (overlay in Figure S5). All pdb structures of 16 macrostates are available in supporting information. 
Comparison of the free energy profile from the two MSM provides a way to ascertain the information generated by the computational protocol. The similarity of the free energies demonstrates that the MSM framework combined with our multi-source seeding strategy is able to discover and similarly rank the key computationally generated conformational variants of c-Abl with or without utilization of c-Abl X-ray structures. The root-meansquared error (RMSE) between the two sets of relative free energies is $1.35 \mathrm{kcal} / \mathrm{mol}$. The mean absolute error (MAE) between the two sets of relative free energies is $1.20 \mathrm{kcal} / \mathrm{mol}$. The median difference between free energies is $0.24 \mathrm{kcal} / \mathrm{mol}$ and $95 \%$ error (frequently used in estimating single data point precision) is $2.4 \mathrm{kcal} / \mathrm{mol}$. Those values indicate this methodology's precision to capture all conformational variants of Abl protein. The free energy values for the 16 macrostates obtained by MSM from Abl and non-Abl starting points are correlated with an $\mathrm{R}^{2}$ of 0.62 (Figure $\mathrm{S6}$ ).

\section{MSM identified a previously publicly unknown Abl conformational state}

To further validate the MSM-derived conformational variability, we first assigned each Abl $\mathrm{X}$-ray structure in the public domain to the closest macrostate based on the all-atom RMSD in the variable region, which consists of the P-loop, A-loop, and aC-helix. Seven out of the 16 macrostates have been observed at least once in the publicly available structures (Figure 3). In addition, we also assigned the Abl structures that are available in the Lilly internal Xray database (not used during the model construction). Interestingly, we identified one macrostate (state 16) that is not present in public structures but exists in the Lilly database where $\mathrm{Abl}$ is in complex with a purine-based ligand WHI-P154 (Figure 4a, see supporting information for experimental details). WHI-P154, originally discovered as JAK3 inhibitor, ${ }^{55}$ has $0.57 \mu \mathrm{M}$ biochemical potency against Abl (as measured by ADP Fluorescence Polarization assay ${ }^{56}$ ). In this complex, interactions are present between the purine nitrogen and the nitrogen of Met318 located in the hinge region, along with the purine/benzoic acid bridging nitrogen and carbonyl of the same methionine. Additionally, the hydroxyl-methyl oxygen forms a water-mediated hydrogen bond with the carbonyl of Arg367 while the benzoic moiety extends partially into the solvent region. The structure has now been deposited in the PDB (id 6BL8).

The Abl X-ray structure in the Lilly internal structural database and its superposition to the reference structure used to define macrostate 16 are shown in Figure $4 \mathrm{~b}$. The high structural similarity is notable, with an RMSD of $0.73 \AA$. Although this conformational state is not populated by publically available structures from the pdb, it displays a similar variable region arrangement to macrostate 4 from the pdb (all non-hydrogen atom RMSD of $2.9 \AA$ in the variable region, see Figure S7). The free energy of macrostate 16 is comparable or lower than some macrostates, which have pdb representation (Figure 2a, S6). We are confident that more predicted MSM-derived conformations can be found in the future, especially for those with low free energies.

Because the reference coordinate set used to define the structural ensemble associated with macrostate 16 was also utilized initially to seed the brute-force MD simulations for the MSM, it is important to evaluate its effectiveness to serve as the centroid of the microstate data cloud within the tessellation cell. To this end, we characterized the spatial distribution 
of the microstates by monitoring the RMSD relative to the reference coordinate set used to define macrostate 16 . The normalized histogram (probability density) of the RMSD, as shown in the supporting information Figure S8, demonstrates the median of the histogram is at $\sim 3.5 \AA$, a distribution similar to that of other macrostates. The majority of the data cloud is near the reference structure serving to define macrostate 16, making it a good representation of the MSM framework. Essentially, the MSM framework indicates that the structural ensemble of macrostate 16 is well defined within the tessellation cell, similar to the other macrostates.

\section{Analysis of the binding pocket of macrostates}

The present computational methodology is more likely to an impact on SBDD efforts if one can exploit the conformational variants to design inhibitors specifically targeting different conformational states. A first step to achieve this goal is to determine if there are significant differences in the shape and overall properties of the binding pocket presented in Abl across the different macrostates. To this end, we compared the shape of the binding pocket of the 16 macrostates using a Tanimoto similarity metric. The latter is correlated with the RMSD of the variable region (Figure S9), however both metrics are unique and can be exploited to describe conformations for different purposes. In Figure 5a the shape of the binding pocket from the different macrostates is compared and the similarity is subjected to Ward clustering. This analysis suggests that macrostate 16 presents the binding pocket that is the most different among all those that have publicly available X-ray structures (the binding pocket of macrostate 12 is actually the most different but there is no available X-ray structure for this conformation). The binding pocket of macrostate 16 is the most similar to that of macrostates 6 and 13 (Tanimoto similarity of 0.9), whereas it is the most dissimilar to that of macrostate 3 (Tanimoto similarity of 0.65). The marked difference in the shape of the binding site of macrostates 16 and 3 can be clearly visualized in Figure 5b. Analysis of binding site shapes can be used for ligand design while variable region conformations might be utilized for designing and understanding of specific protein-protein interactions.

\section{Conformation variability of the P-loop}

The conformational variability of the P-loop and its impact on improving the selectivity profile has long been of interest to SBDD. ${ }^{57}$ The P-loop in the majority of protein kinase structures displays an "extended" conformation, while a less common "kinked" (partially folded) conformation has been observed in some protein kinases such as c-Abl, c-Met, and Aurora A kinase. ${ }^{57-58}$ In the case of Abl kinase, it has been suggested that the kinked conformation is crucial for the selectivity of imatinib toward c-Abl rather than c-Src. ${ }^{58-59}$ Nonetheless, the conformational variability of the P-loop region and the associated propensities in a protein kinase have not been systematically analyzed, even for c-Abl.

We first analyzed the P-loop conformations in the X-ray crystallographic structures of c-Abl that were obtained from the PDB (80 separate X-ray structures from 46 unique PDB IDs). A cluster analysis of the conformations of the P-loop was carried out using an agglomerative hierarchical method with Ward linkage. Inspection of the dendrogram from the cluster analysis (displayed in Figure S10) suggests that four distinct conformations of the P-loop exist (the representative structures are displayed in Figure 1). To evaluate the propensity of 
the conformations, a two-dimensional free energy landscape (a.k.a. 2D-PMF) was constructed for the P-loop motif based on the MSMs using a previously established protocol. 43 The same two distances as the features to describe P-loop conformational flexibility for constructing the MSMs were utilized as the reaction coordinates of the 2D-PMF. The conformational free energy landscape of the P-loop (Figure 6a) shows that the P-loop of cAbl can indeed adopt multiple stable conformations, as indicated by the $\mathrm{X}$-ray structures, even in the apo-kinase form. Remarkably, the free energy landscape also indicates that the extended conformation is the most thermodynamically favorable one for the P-loop in the apo c-Abl kinase, even though the majority of the c-Abl X-ray structures display the kinked conformation. This observation suggests that analysis of the propensity of conformational variants observed in X-ray structures, which would predict a different result in the case of cAbl, could be potentially unreliable and even misleading. The power of the MSM-based analysis is further highlighted by the fact that a novel conformation of the P-loop not previously observed in publicly available X-ray structures is revealed by the computed free energy landscape.

\section{Thermodynamics and kinetics of the DFG-flip}

$\mathrm{X}$-ray crystallographic structures of c-Abl in complex with Gleevec display a particular conformation called the "DFG-out" in which the DFG motif is flipped almost $180^{\circ}$ relative to the standard "DFG-in" active conformation. In addition to accommodating type II protein kinase inhibitors, the DFG-out conformation is also an important feature regarding kinase regulation, as it disrupts the ability for ATP-binding and disassembles the R-spine needed for catalysis. Because of its great importance, the conformation of the DFG motif and its impact on the binding specificity of inhibitors has been the subject of many computational studies ${ }^{60-62}$ Generally, the results from computations support the concept of a thermodynamic penalty for the DFG flip ${ }^{60-61}$. The thermodynamic penalty view is also supported by a structural propensity analysis of protein kinase using sequence-based methods. ${ }^{62}$ As shown previously, the DFG-in and the DFG-out states both correspond to an ensemble of structures (i.e. there is an intrinsic conformational variability within the DFGin/-out states). The issue of Gleevec binding and DFG-flip in Src kinase was recently investigated by Morando et al., utilizing a combination of experimental and computational methods. ${ }^{63}$ NMR, surface plasmon resonance (SPR) spectroscopy, and metadynamics simulations were performed. Both conformational selection and induced-fit mechanisms were suggested to play a role in Gleevec binding to c-Src kinase. This study also revealed the importance of temperature in the kinetics of Gleevec binding. Furthermore, induced-fit effect in the binding of Gleevec to WT and T315I Abl kinase has also been investigated by Lovera et al. ${ }^{64}$ Extensive deviations in the conformational free energy landscape of the DFG-flip and activation-loop dynamics were discovered between the WT and the Gleevecresistant mutant. ${ }^{64}$ The T315I mutation was also shown to influence the kinetics of binding.

The free energy landscape of the DFG-flip based on our MSMs provides us with a sharper view of the thermodynamics underlying this transition by incorporating the intrinsic conformational variability within the DFG-in/-out states. The 2D-PMF in Figure 6b shows unambiguously that the DFG-in conformation is more favorable than the DFG-out conformation, confirming that the thermodynamic penalty view is valid. As reflected by the 
free energy landscape, the DFG-out conformation is a stable state, albeit slightly less favorable than DFG-in. For Abl kinase, the conformational selection mechanism plays a role in the binding of type-II kinase inhibitors, meaning that there are spontaneous occurrences of the DFG-out(apo) conformations ready to bind type-II ligands. Interestingly, the X-ray structure of the Abl-imatinib complex (PDB id: $1 \mathrm{IEP}^{65}$ ) is not at the bottom of a local minimum in the calculated free energy landscape shown in Figure 6b. Some additional structural changes akin to induced-fit effects are required to adopt the observed "imatinibbound DFG-out" conformation. This exemplifies the importance of conformational selection combined with more subtle induced-fit effects in the binding of type-II kinase selective inhibitors. Another feature revealed by the calculated free energy landscape of the DFG motif is the existence of a metastable state ( $d_{3}=7$ and $d_{4}=17$ on Figure $6 \mathrm{~b}$ ). In this metastable state, F382 has moved out of the deep hydrophobic pocket but has not yet swapped its position with D381, suggesting that F382 is a more flexible element than D381.

Accordingly, displacement of D381 could present a kinetic bottleneck needed to finalize the DFG flip. As will be discussed below, occurrence of the metastable state appears to be coupled with a specific conformation of the aC-helix and thus, may offer binding site for non-ATP competitive inhibitors (type-III kinase inhibitors). One should note that the aspartate residue 381 in the DFG-motif is deprotonated in the present simulations. The thermodynamics as well as the kinetics of the DFG flip is likely to be affected by the protonation of Asp381, as suggested by previous work. ${ }^{66-67}$

The overall picture of the conformational landscape of c-Abl KD and c-Src KD are in qualitative agreement: the DFG-in inactive state is indeed thermodynamically the most stable state. In Src/CDK systems, the inactive KD conformation also corresponds to the DFG-in states. The thermodynamic penalty view does not imply that the active form of c$\mathrm{Abl} \mathrm{KD}$ is more stable than the inactive form. In fact, as illustrated in Figure 2, the Src/CDK inactive state (or more generally, the DFG-in inactive state) has a lower free energy than that of the active state. This consistency also suggests the features of the KD conformational landscape is conserved across tyrosine kinases.

The kinetics of DFG-flip has long been recognized to influence the binding of type II protein kinase inhibitors, as showcased in Gleevec binding. ${ }^{68}$ However, unraveling the kinetics of DFG-flip remains challenging for both experimental and computational studies. An earlier study by Vogtherr et al. on the DFG-flip of p38a MAPK has demonstrated that the transition takes place on a millisecond time scale, as measured by nuclear magnetic resonance (NMR) spectroscopy ${ }^{69}$. More recently, Kern and co-workers have argued that the origin of Gleevec's binding specificity lies predominantly in a slow conformational change occurring after binding: they revisited the issue of binding of Gleevec in c-Abl and c-Src using NMR and stop-flow fluorescence experiments ${ }^{70}$. The millisecond transition time scale poses great challenge on obtaining kinetic information with modeling. However, the framework of MSM provides its advantage in obtaining longer time-scale from relatively short time-scale MD simulations: one can propagate the master equation to generate a time series of MSM microstates at any length. Furthermore, for each MSM microstate, one can sample the corresponding structural ensemble so that structural information can be utilized. An example of such "kinetic" trajectory is illustrated in estimating the activation time-scale of c-Src KD. 43 To reflect the time-scale of the DFG-flip in c-Abl, we constructed two such "kinetic" 
trajectories of 1 and 10 milliseconds respectively (displayed in Figure 7). Both kinetic trajectories were based on the MSM consisting of 10,000 microstates and having a lag-time of 40 ns. The distance between $\mathrm{C} \gamma$ of F382 and Ca of I293 was utilized to gauge the DFG flip, as highlighted in the right panel of Figure 7. As demonstrated in both plots, our MD simulations also suggest that the transition of the DFG-motif in the unphosphorylated apoAbl kinase occurs in the millisecond time-scale.

\section{Conformation variability of the aC-helix and A-loop}

A good example of the conformational variability of the aC-helix and the A-loop is given by the Src/CDK-like inactive state. ${ }^{61}$ The structural characteristics of the Src/CDK inactive state are the outward rotation of the aC-helix (so that a critical salt-bridge for catalysis is broken), and the partially folded A-loop. A structurally similar inactive state has also been observed in epidermal growth factor receptor (EGFR). ${ }^{71}$ One should note that the DFGmotif displays the "in" conformation in CDK, Src-family kinases, and EGFR when they adopt the Src/CDK-like inactive state. The existence of multiple inactive states illustrates the diversity in down-regulating catalytic activity in protein tyrosine kinase.

To characterize the conformational variability of the aC-helix and the A-loop, we project the MSM microstates onto two structural features that resemble those we utilized in the study of c-Src ${ }^{34,35}$. We first categorized our data based on the conformational state of the DFG-motif. The subset that is in the DFG-in state corresponds to our previous work on c-Src. ${ }^{61}$ However, unlike our previous work on c-Src in which the conformational sampling was focused on the vicinity of the transition pathways optimized by the string method with swarms-of-trajectories, the sampling in c-Abl was extended to a much broader conformational space which can be reflected in the 2D-PMF (Figure 6c). The 2D-PMF of the aC-helix and the A-loop demonstrates certain degree of similarity to that of the c-Src: there are multiple stable metastates to activate c-Abl, and the catalytically competent state is energetically unfavorable when the A-loop is unphosphorylated. However, the 2D-PMF of cAbl also demonstrates an expanded "intermediate" region when compared with the one of cSrc. This behavior was also observed in the recent study of the apo-BTK kinase domain by MD and MSM. ${ }^{20}$ This consistency in Src, Abl and BTK suggested the importance of the Src-like inactive state in regulating tyrosine kinase activity.

Finally, we also reconstructed the 2D-PMF of the aC-helix and the A-loop with the same two collective variables, utilizing the DFG-out subset. The result is shown in Figure 6d. Compared with the free energy landscape of Figure 6c (DFG-in), the 2D-PMF in Figure 6d displays an increased movement of the aC-helix. One should note that the metastable state appeared in the 2D-PMF of the DFG motif is included in the DFG-out subset. The free energy basin located at the lower-right corner of Figure $6 \mathrm{~d}$ contains that metastable state. Essentially, F382 is pushed toward the ATP-binding site, and the conserved catalytically crucial Lys-Glu salt-bridge is not formed as the glutamate residue is still pointing outward. This particular arrangement of the DFG motif and the aC-helix has created a pocket that may be leveraged by non-ATP competitive inhibitors. 


\section{Allosteric coupling between the myristoyl and ATP binding sites}

Crystal structures of the multidomain kinase core region of c-Abl in the autoinhibited state revealed a myristoyl-binding pocket at the C-terminus of the KD. ${ }^{72-73}$ The aI-helix, which is straight in the activated state, adopts a bent conformation when the myristoyl group is present in the binding pocket. It is thought that the bent in the aI-helix enables the Srchomology 2 (SH2) domain to interact with the kinase domain to contribute in the stabilization of the autoinhibited state. ${ }^{34,72-73}$ These findings lead to the discovery of allosteric inhibitors that occupy the myristoyl-binding pocket to stabilize the autoinhibited state. ${ }^{74-75}$ To explore the allosteric coupling between the conformation of the myristolbinding site and the DFG motif at the ATP binding site, we projected the MSM microstates onto the distance d4 (used to monitor the DFG flip in Fig. 6b) and the distance between I432 and L341 (d8) monitoring the opening of the myristol-binding site (I432 is part of the Cspine). The 2D-PMF characterized in terms of these two order parameters is shown in Figure $6 e$. The free energy landscape shows that the myristoyl-binding pocket becomes tight when the DFG motif adopts an "in" conformation (and hence reducing the binding of the myristoyl group), while the DFG-out state tends to possess a larger myristoyl-binding pocket (able to bind the myristoyl group). Even though the MD simulations were generated for the Apo state of the KD, there is sufficient information from the MSM to explain the allosteric coupling of the two binding sites from an energetic point of view. While large-scale motions can be observed for the myristoyl-binding pocket regardless of the conformation of the DFG motif, the free energy cost to expand the myristoyl-binding pocket is lower when the DFGmotif is in the "in" state. In other words, the myristoyl-binding pocket is more likely to expand in the DFG-out state than in the DFG-in state. Since expanding the myristoylbinding pocket can lead to disrupting of the interaction between SH2 and the kinase domain, stabilizing the DFG-out state with imatinib can lead to an increased level of the disassembled core region. This behavior is consistent with what has been observed by NMR experiment: binding of imatinib lead to an unexpected opening of the multidomain kinase core region. ${ }^{34}$

\section{SUMMARY}

The present effort demonstrates the value of a computational strategy based on MSM to characterize the conformational variants of a target protein of interest. Our motivation to develop and validate this strategy stems directly from the situation confronting molecular modeling in the context of a structure-based drug discovery effort in the pharmaceutical industry. To the question whether a MSM-based computational strategy is able to accurately predict and rank-order the conformational variants of a target protein of interest, this work answers a resounding "yes". Importantly, the present work also shows that de novo alternative structures of uncharacterized kinases can effectively be discovered successfully by sampling the apo-kinase, even when no X-ray structures are available. The analysis can also reveal the underlying allosteric coupling between the myristoyl and ATP binding sites.

The computational strategy is specifically designed to overcome the common challenges of conformational sampling by exploiting one of the major strengths of the MSM approach: the information content derived from the MSM framework is driven by counting "state-to-state 
transitions" rather than simply by counting "occurrences of a state" as in traditional equilibrium sampling. Thus, it does not matter how many simulations started from a given state are accumulated in the aggregate data, the MSM naturally yields the unbiased equilibrium probability from the transition matrix. Furthermore, this means that a variety of methods can be used to generate plausible starting configurations while keeping the results free of biases. The matching free energies (red and blue curves, Figure 2) demonstrate quantitatively that the multi-source seeding strategy is able to effectively discover and rank all the key conformational variants of c-Abl/Abl1, even without a priori knowledge of Abl $\mathrm{X}$-ray structures. This result is very encouraging and generally indicative of the overall robustness of the computational method.

While the present results were obtained by considering only the catalytic kinase domain, it will ultimately be important to consider the conformational variants in the context of the multi-domain kinase and phosphorylation to maximize the impact of this type of information in the context of a structure-based effort in the pharmaceutical industry. ${ }^{76-78}$ On the other hand, the current application of the multi-source seeding strategy on the conformational states of Abl kinase domain seems to suggest that the known states of this target are mostly accessible via conformational selection rather than substantial induced-fit effect. In future efforts, the computational protocol will be further tested and validated based on the conformational plasticity of other kinases or protein targets.

\section{Supplementary Material}

Refer to Web version on PubMed Central for supplementary material.

\section{Acknowledgments}

This work is supported by the National Cancer Institute (NCI) of the National Institutes of Health (NIH) through grant R01-CAO93577. The computations are supported in part by NIH through resources provided by the Computation Institute and the Biological Sciences Division of the University of Chicago and Argonne National Laboratory under grant S10 RR029030-01, by the Extreme Science and Engineering Discovery Environment (XSEDE) grant number OCI-1053575, and by the University of Chicago Research Computing Center. The authors gratefully acknowledge Hal Lewis for an initial structure and maps. This research used resources of the Advanced Photon Source, a U.S. Department of Energy (DOE) Office of Science User Facility operated for the DOE Office of Science by Argonne National Laboratory under Contract No. DE-AC02-06CH11357. Use of the Lilly Research Laboratories Collaborative Access Team (LRL-CAT) beamline at Sector 31 of the Advanced Photon Source was provided by Eli Lilly Company, which operates the facility.

\section{References}

1. Shoichet BK. Virtual screening of chemical libraries. Nature. 2004; 432(7019):862-5. [PubMed: 15602552]

2. Leach AR, Shoichet BK, Peishoff CE. Prediction of protein-ligand interactions. Docking and scoring: successes and gaps. J Med Chem. 2006; 49(20):5851-5. [PubMed: 17004700]

3. Ghosh S, Nie A, An J, Huang Z. Structure-based virtual screening of chemical libraries for drug discovery. Curr Opin Chem Biol. 2006; 10(3):194-202. [PubMed: 16675286]

4. Green DV. Virtual screening of chemical libraries for drug discovery. Expert Opin Drug Discov. 2008; 3(9):1011-26. [PubMed: 23506177]

5. Keiser MJ, Irwin JJ, Shoichet BK. The chemical basis of pharmacology. Biochemistry. 2010; 49(48):10267-76. [PubMed: 21058655] 
6. Ma XH, Zhu F, Liu X, Shi Z, Zhang JX, Yang SY, Wei YQ, Chen YZ. Virtual screening methods as tools for drug lead discovery from large chemical libraries. Current medicinal chemistry. 2012; 19(32):5562-71. [PubMed: 23016548]

7. Manning G, Whyte DB, Martinez R, Hunter T, Sudarsanam S. The protein kinase complement of the human genome. Science. 2002; 298(5600):1912-1934. [PubMed: 12471243]

8. Fasano M, Della Corte CM, Califano R, Capuano A, Troiani T, Martinelli E, Ciardiello F, Morgillo F. Type III or allosteric kinase inhibitors for the treatment of non-small cell lung cancer. Expert Opin Investig Drugs. 2014; 23(6):809-21.

9. Bowman GR, Huang X, Pande VS. Using generalized ensemble simulations and Markov state models to identify conformational states. Methods. 2009; 49(2):197-201. [PubMed: 19410002]

10. Pande VS, Beauchamp K, Bowman GR. Everything you wanted to know about Markov State Models but were afraid to ask. Methods. 2010; 52(1):99-105. [PubMed: 20570730]

11. Prinz JH, Wu H, Sarich M, Keller B, Senne M, Held M, Chodera JD, Schutte C, Noe F. Markov models of molecular kinetics: generation and validation. J Chem Phys. 2011; 134(17):174105. [PubMed: 21548671]

12. Bowman, GR, Pande, VS, Noé, F. An Introduction to Markov State Models and Their Application to Long Timescale Molecular Simulation. Vol. 797. Springer; Dordrecht: 2014.

13. Huang X, Bowman GR, Pande VS. Convergence of folding free energy landscapes via application of enhanced sampling methods in a distributed computing environment. J Chem Phys. 2008; 128(20):205106. [PubMed: 18513049]

14. Buch I, Giorgino T, De Fabritiis G. Complete reconstruction of an enzyme-inhibitor binding process by molecular dynamics simulations. Proc Natl Acad Sci USA. 2011; 108(25):1018410189. [PubMed: 21646537]

15. Kohlhoff KJ, Shukla D, Lawrenz M, Bowman GR, Konerding DE, Belov D, Altman RB, Pande VS. Cloud-based simulations on Google Exacycle reveal ligand modulation of GPCR activation pathways. Nat Chem. 2014; 6(1):15-21. [PubMed: 24345941]

16. Plattner N, Noe F. Protein conformational plasticity and complex ligand-binding kinetics explored by atomistic simulations and Markov models. Nat Commun. 2015; 6:7653. [PubMed: 26134632]

17. Malmstrom RD, Kornev AP, Taylor SS, Amaro RE. Allostery through the computational microscope: cAMP activation of a canonical signalling domain. Nat Commun. 2015; 6:7588. [PubMed: 26145448]

18. Da LT, Pardo-Avila F, Xu L, Silva DA, Zhang L, Gao X, Wang D, Huang X. Bridge helix bending promotes RNA polymerase II backtracking through a critical and conserved threonine residue. Nat Commun. 2016; 7:11244. [PubMed: 27091704]

19. Plattner N, Doerr S, De Fabritiis G, Noé F. Complete protein-protein association kinetics in atomic detail revealed by molecular dynamics simulations and Markov modelling. Nat Chem. 2017; 9:1005. [PubMed: 28937668]

20. Sultan MM, Denny RA, Unwalla R, Lovering F, Pande VS. Millisecond dynamics of BTK reveal kinome-wide conformational plasticity within the apo kinase domain. Sci Rep. 2017; 7(1):15604. [PubMed: 29142210]

21. Harrigan MP, McKiernan KA, Shanmugasundaram V, Denny RA, Pande VS. Markov modeling reveals novel intracellular modulation of the human TREK-2 selectivity filter. Sci Rep. 2017; 7(1): 632. [PubMed: 28377596]

22. Chodera JD, Singhal N, Pande VS, Dill KA, Swope WC. Automatic discovery of metastable states for the construction of Markov models of macromolecular conformational dynamics. J Chem Phys. 2007; 126(15):155101. [PubMed: 17461665]

23. Bowman GR, Beauchamp KA, Boxer G, Pande VS. Progress and challenges in the automated construction of Markov state models for full protein systems. J Chem Phys. 2009; 131(12):124101. [PubMed: 19791846]

24. Cronkite-Ratcliff B, Pande V. MSMExplorer: visualizing Markov state models for biomolecule folding simulations. Bioinformatics. 2013; 29(7):950-2. [PubMed: 23365411]

25. Bowman GR, Ensign DL, Pande VS. Enhanced modeling via network theory: Adaptive sampling of Markov state models. J Chem Theory Comput. 2010; 6(3):787-794. [PubMed: 23626502] 
26. Pan AC, Roux B. Building Markov state models along pathways to determine free energies and rates of transitions. J Chem Phys. 2008; 129(6):064107. [PubMed: 18715051]

27. Pan AC, Sezer D, Roux B. Finding transition pathways using the string method with swarms of trajectories. J Phys Chem B. 2008; 112(11):3432-40. [PubMed: 18290641]

28. Woo HJ, Roux B. Calculation of absolute protein-ligand binding free energy from computer simulations. Proc Natl Acad Sci USA. 2005; 102(19):6825-6830. [PubMed: 15867154]

29. Gumbart JC, Roux B, Chipot C. Standard binding free energies from computer simulations: What is the best strategy? J Chem Theory Comput. 2013; 9(1):794-802. [PubMed: 23794960]

30. Mey ASJS, Wu H, Noe F. xTRAM: Estimating Equilibrium Expectations from Time-Correlated Simulation Data at Multiple Thermodynamic States. Phys Rev X. 2014; 4:041018.

31. Webb, B, Sali, A. Curr Protoc Bioinformatics. John Wiley \& Sons, Inc; 2002. Comparative Protein Structure Modeling Using MODELLER; 5.6.1-5.6.32.

32. Schlitter J, Engels M, Krüger P. Targeted molecular dynamics: A new approach for searching pathways of conformational transitions. J Mol Graph. 1994; 12(2):84-89. [PubMed: 7918256]

33. Das R, Baker D. Macromolecular modeling with rosetta. Annu Rev Biochem. 2008; 77:363-82. [PubMed: 18410248]

34. Panjarian S, Iacob RE, Chen SG, Engen JR, Smithgall TE. Structure and Dynamic Regulation of Abl Kinases. J Biol Chem. 2013; 288(8):5443-5450. [PubMed: 23316053]

35. Fiser A, Do RKG, Šali A. Modeling of loops in protein structures. Protein Sci. 2000; 9(9):17531773. [PubMed: 11045621]

36. Case, DA, Babin, V, Berryman, JT, Betz, RM, Cai, Q, Cerutti, DS, Cheatham, ITE, Darden, TA, Duke, RE, Gohlke, H, Goetz, AW, Gusarov, S, Homeyer, N, Janowski, P, Kaus, J, Kolossváry, I, Kovalenko, A, Lee, TS, LeGrand, S, Luchko, T, Luo, R, Madej, B, Merz, KM, Paesani, F, Roe, DR, Roitberg, AE, Sagui, C, Salomon-Ferrer, R, Seabra, G, Simmerling, CL, Smith, W, Swails, J, Walker, RC, Wang, J, Wolf, RM, Wu, X, Kollman, PA. AMBER 14. University of California; San Francisco: 2014.

37. Salomon-Ferrer R, Götz AW, Poole D, Le Grand S, Walker RC. Routine Microsecond Molecular Dynamics Simulations with AMBER on GPUs. 2. Explicit Solvent Particle Mesh Ewald. J Chem Theory Comput. 2013; 9(9):3878-3888. [PubMed: 26592383]

38. Maier JA, Martinez C, Kasavajhala K, Wickstrom L, Hauser KE, Simmerling C. ff14SB: Improving the Accuracy of Protein Side Chain and Backbone Parameters from ff99SB. J Chem Theory Comput. 2015; 11(8):3696-3713. [PubMed: 26574453]

39. Jorgensen WL, Chandrasekhar J, Madura JD, Impey RW, Klein ML. Comparison of Simple Potential Functions for Simulating Liquid Water. J Chem Phys. 1983; 79(2):926-935.

40. Berendsen HJC, Postma JPM, van Gunsteren WF, DiNola A, Haak JR. Molecular dynamics with coupling to an external bath. J Chem Phys. 1984; 81(8):3684-3690.

41. Darden T, York D, Pedersen L. Particle Mesh Ewald - an N.Log(N) Method for Ewald Sums in Large Systems. J Chem Phys. 1993; 98(12):10089-10092.

42. Ryckaert JP, Ciccotti G, Berendsen HJC. Numerical-Integration of Cartesian Equations of Motion of a System with Constraints - Molecular-Dynamics of N-Alkanes. J Comput Phys. 1977; 23(3): 327-341.

43. Shukla D, Meng Y, Roux B, Pande VS. Activation pathway of Src kinase reveals intermediate states as targets for drug design. Nat Commun. 2014; 5:3397. [PubMed: 24584478]

44. Zimmerman MI, Bowman GR. FAST Conformational Searches by Balancing Exploration/ Exploitation Trade-Offs. J Chem Theory Comput. 2015; 11(12):5747-57. [PubMed: 26588361]

45. Harrigan MP, Sultan MM, Hernandez CX, Husic BE, Eastman P, Schwantes CR, Beauchamp KA, McGibbon RT, Pande VS. MSMBuilder: Statistical Models for Biomolecular Dynamics. Biophys J. 2017; 112(1):10-15. [PubMed: 28076801]

46. Noe F, Clementi C. Kinetic Distance and Kinetic Maps from Molecular Dynamics Simulation. J Chem Theory Comput. 2015; 11(10):5002-11. [PubMed: 26574285]

47. Perez-Hernandez G, Paul F, Giorgino T, De Fabritiis G, Noe F. Identification of slow molecular order parameters for Markov model construction. J Chem Phys. 2013; 139(1):015102. [PubMed: 23822324] 
48. Schwantes CR, Pande VS. Improvements in Markov State Model Construction Reveal Many NonNative Interactions in the Folding of NTL9. J Chem Theory Comput. 2013; 9(4):2000-2009. [PubMed: 23750122]

49. Sculley, DM. Web-Scale K-Means Clustering. Proceedings of the 19th international conference on World wide web; Raleigh, North Carolina, USA: ACM; 2010. 1177-1178.

50. Noé F, Nüske F. A Variational Approach to Modeling Slow Processes in Stochastic Dynamical Systems. Multiscale Model Simul. 2013; 11(2):635-655.

51. Nüske F, Keller BG, Pérez-Hernández G, Mey ASJS, Noé F. Variational Approach to Molecular Kinetics. J Chem Theory Comput. 2014; 10(4):1739-1752. [PubMed: 26580382]

52. Gao C, Desaphy J, Vieth M. Are induced fit protein conformational changes caused by ligandbinding predictable? A molecular dynamics investigation. J Comput Chem. 2017; 38(15):12291237. [PubMed: 28419481]

53. Cowan-Jacob SW, Fendrich G, Floersheimer A, Furet P, Liebetanz J, Rummel G, Rheinberger P, Centeleghe M, Fabbro D, Manley PW. Structural biology contributions to the discovery of drugs to treat chronic myelogenous leukaemia. Acta Crystallogr D. 2007; 63:80-93. [PubMed: 17164530]

54. MOE. Molecular Operating Environment (MOE), 2016.08. Chemical Computing Group ULC; 1010 Sherbooke St. West, Suite \#910, Montreal, QC, Canada, H3A 2R7: 2017.

55. Sudbeck EA, Liu X-P, Narla RK, Mahajan S, Ghosh S, Mao C, Uckun FM. Structure-based Design of Specific Inhibitors of Janus Kinase 3 as Apoptosis-inducing Antileukemic Agents. Clin Cancer Res. 1999; 5(6):1569-1582. [PubMed: 10389946]

56. Sutherland JJ, Higgs RE, Watson I, Vieth M. Chemical Fragments as Foundations for Understanding Target Space and Activity Prediction. J Med Chem. 2008; 51(9):2689-2700. [PubMed: 18386916]

57. Guimaraes CRW, Rai BK, Munchhof MJ, Liu SP, Wang J, Bhattacharya SK, Buckbinder L. Understanding the Impact of the P-loop Conformation on Kinase Selectivity. J Chem Inf Model. 2011; 51(6):1199-1204. [PubMed: 21568278]

58. Seeliger MA, Ranjitkar P, Kasap C, Shan YB, Shaw DE, Shah NP, Kuriyan J, Maly DJ. Equally Potent Inhibition of c-Src and Abl by Compounds that Recognize Inactive Kinase Conformations. Cancer Res. 2009; 69(6):2384-2392. [PubMed: 19276351]

59. Seeliger MA, Nagar B, Frank F, Cao X, Henderson MN, Kuriyan J. c-Src binds to the cancer drug imatinib with an inactive Abl/c-Kit conformation and a distributed thermodynamic penalty. Structure. 2007; 15(3):299-311. [PubMed: 17355866]

60. Lovera S, Sutto L, Boubeva R, Scapozza L, Dolker N, Gervasio FL. The Different Flexibility of cSrc and c-Abl Kinases Regulates the Accessibility of a Druggable Inactive Conformation. J Am Chem Soc. 2012; 134(5):2496-2499. [PubMed: 22280319]

61. Meng Y, Pond MP, Roux B. Tyrosine Kinase Activation and Conformational Flexibility: Lessons from Src-Family Tyrosine Kinases. Acc Chem Res. 2017; 50(5):1193-1201. [PubMed: 28426203]

62. Haldane A, Flynn WF, He P, Vijayan RSK, Levy RM. Structural propensities of kinase family proteins from a Potts model of residue co-variation. Protein Sci. 2016; 25(8):1378-1384. [PubMed: 27241634]

63. Morando MA, Saladino G, D’Amelio N, Pucheta-Martinez E, Lovera S, Lelli M, Lopez-Mendez B, Marenchino M, Campos-Olivas R, Gervasio FL. Conformational Selection and Induced Fit Mechanisms in the Binding of an Anticancer Drug to the c-Src Kinase. Sci Rep. 2016; 6:24439. [PubMed: 27087366]

64. Lovera S, Morando M, Pucheta-Martinez E, Martinez-Torrecuadrada JL, Saladino G, Gervasio FL. Towards a Molecular Understanding of the Link between Imatinib Resistance and Kinase Conformational Dynamics. PLoS Comput Biol. 2015; 11(11):e1004578. [PubMed: 26606374]

65. Schindler T, Bornmann W, Pellicena P, Miller WT, Clarkson B, Kuriyan J. Structural mechanism for STI-571 inhibition of Abelson tyrosine kinase. Science. 2000; 289(5486):1938-1942. [PubMed: 10988075]

66. Shan YB, Seeliger MA, Eastwood MP, Frank F, Xu HF, Jensen MO, Dror RO, Kuriyan J, Shaw DE. A conserved protonation-dependent switch controls drug binding in the Abl kinase. Proc Natl Acad Sci U S A. 2009; 106(1):139-144. [PubMed: 19109437] 
67. Meng Y, Lin YL, Roux B. Computational study of the "DFG-flip" conformational transition in cAbl and c-Src tyrosine kinases. J Phys Chem B. 2015; 119(4):1443-56. [PubMed: 25548962]

68. Vajpai N, Strauss A, Fendrich G, Cowan-Jacob SW, Manley PW, Grzesiek S, Jahnke W. Solution conformations and dynamics of ABL kinase-inhibitor complexes determined by NMR substantiate the different binding modes of imatinib/nilotinib and dasatinib. J Biol Chem. 2008; 283(26): 18292-18302. [PubMed: 18434310]

69. Vogtherr M, Saxena K, Hoelder S, Grimme S, Betz M, Schieborr U, Pescatore B, Robin M, Delarbre L, Langer T, Wendt KU, Schwalbe H. NMR characterization of kinase p38 dynamics in free and ligand-bound forms. Angew Chem Int Ed Engl. 2006; 45(6):993-997. [PubMed: 16374788]

70. Agafonov RV, Wilson C, Otten R, Buosi V, Kern D. Energetic dissection of Gleevec's selectivity toward human tyrosine kinases. Nat Struct Mol Biol. 2014; 21(10):848-853. [PubMed: 25218445]

71. Jura N, Zhang XW, Endres NF, Seeliger MA, Schindler T, Kuriyan J. Catalytic Control in the EGF Receptor and Its Connection to General Kinase Regulatory Mechanisms. Mol Cell. 2011; 42(1):922. [PubMed: 21474065]

72. Hantschel O, Nagar B, Guettler S, Kretzschmar J, Dorey K, Kuriyan J, Superti-Furga G. A myristoyl/phosphotyrosine switch regulates c-Abl. Cell. 2003; 112(6):845-857. [PubMed: 12654250]

73. Nagar B, Hantschel O, Young MA, Scheffzek K, Veach D, Bornmann V, Clarkson B, Superti-Furga G, Kuriyan J. Structural basis for the autoinhibition of c-Abl tyrosine kinase. Cell. 2003; 112(6): 859-871. [PubMed: 12654251]

74. Iacob RE, Zhang JM, Gray NS, Engen JR. Allosteric Interactions between the Myristate- and ATPSite of the Abl Kinase. PLoS One. 2011; 6(1):e15929. [PubMed: 21264348]

75. Zhang JM, Adrian FJ, Jahnke W, Cowan-Jacob SW, Li AG, Iacob RE, Sim T, Powers J, Dierks C, Sun FX, Guo GR, Ding Q, Okram B, Choi Y, Wojciechowski A, Deng XM, Liu GX, Fendrich G, Strauss A, Vajpai N, Grzesiek S, Tuntland T, Liu Y, Bursulaya B, Azam M, Manley PW, Engen JR, Daley GQ, Warmuth M, Gray NS. Targeting Bcr-Abl by combining allosteric with ATP-bindingsite inhibitors. Nature. 2010; 463(7280):501-U116. [PubMed: 20072125]

76. Faraldo-Gomez JD, Roux B. On the importance of a funneled energy landscape for the assembly and regulation of multidomain Src tyrosine kinases. Proc Natl Acad Sci USA. 2007; 104(34): 13643-8. [PubMed: 17699616]

77. Meng Y, Roux B. Locking the Active Conformation of c-Src Kinase through the Phosphorylation of the Activation Loop. J Mol Biol. 2014; 426(2):423-435. [PubMed: 24103328]

78. Fajer M, Meng Y, Roux B. The Activation of c-Src Tyrosine Kinase: Conformational Transition Pathway and Free Energy Landscape. J Phys Chem B. 2016; 121(15):3352-3363. [PubMed: 27715044] 


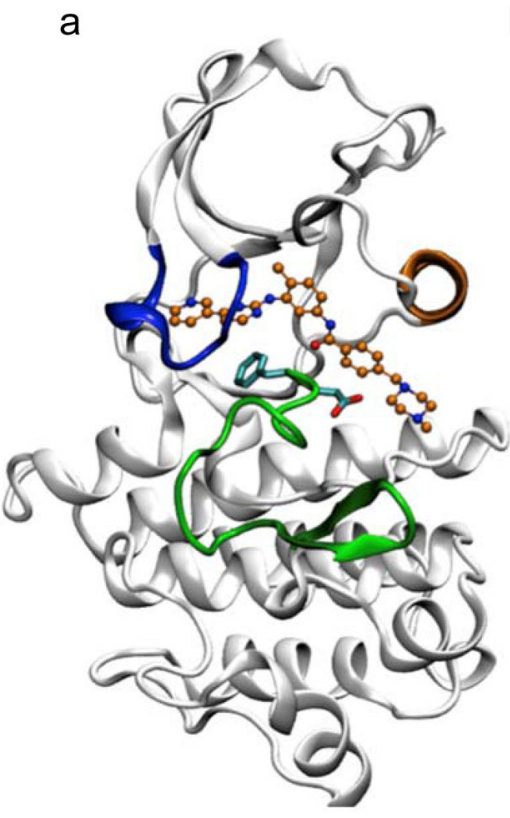

b
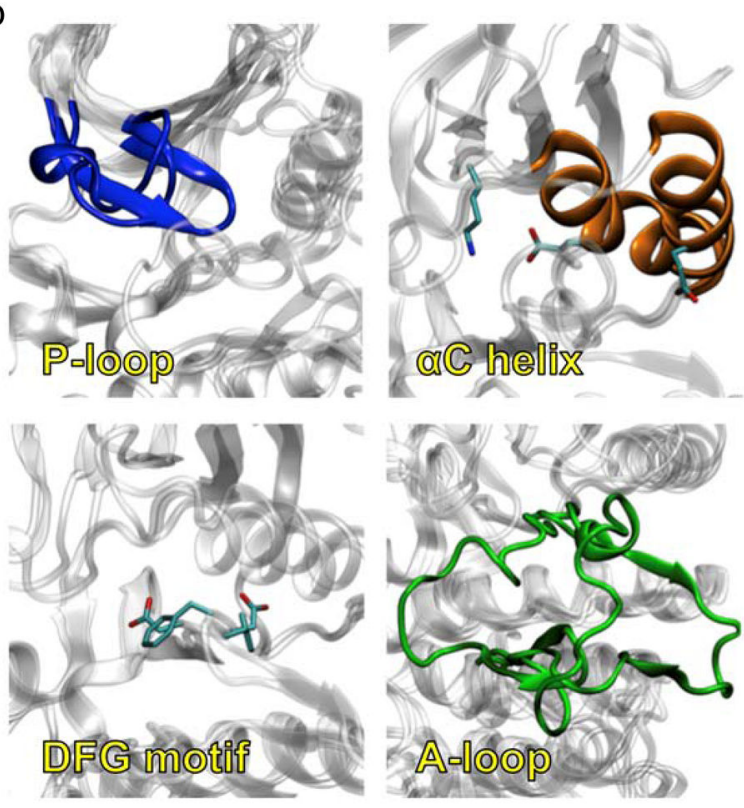

Figure 1.

c-Abl structures and the conformational variability in the kinase domain. (a) Cartoon representation of the kinase domain (KD) of c-Abl (based on PDB id: 1IEP). (b) Conformational variations displayed in the c-Abl KD. 

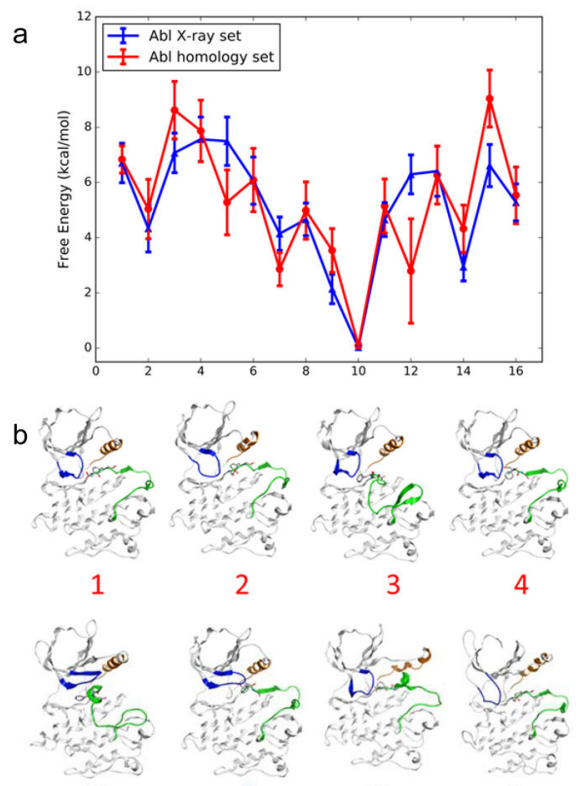

6

7

8
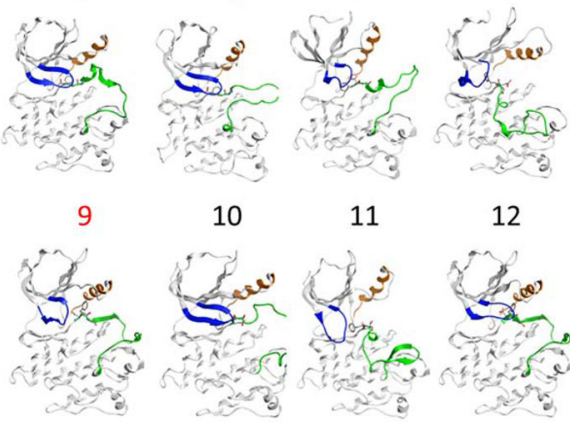

10

11

12

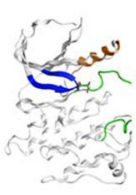

14

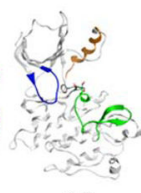

15

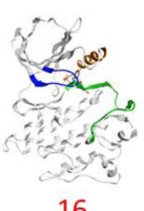

16

Figure 2.

Macrostates mapping the conformational variants of the kinase domain. (a) Free energy profile as a function of the macrostate index. The root-mean-squared error (RMSE) between the two sets is $1.35 \mathrm{kcal} / \mathrm{mol}$, and the mean absolute error (MAE) between the two sets of is $1.2 \mathrm{kcal} / \mathrm{mol}$. Error bars are shown for each set. Significant deviations between the two sets of relative free energies occur at macrostate 5, 12, and 15, while a relatively large deviation also occurs at macrostate 3. At state 5, a "Frankenstein" state was added to the reference set simulation, while a "Frankenstein" state was added to the blind-test set simulations for state 12 and 15 respectively indicating the starting structure generation with this approach given current sampling regime can lead to lower precision. The relatively poor convergence at macrostate 3 is likely caused by lack of sampling in the homology set. (b) Reference structures used to define the ensemble associated with all 16 macrostates (the red number indicate that at least one $\mathrm{X}$-ray structure is available). aC Helix ribbon is colored orange, $\mathrm{P}$ Loop in blue and activation loop in green. DFG motif residues are shown as sticks. 


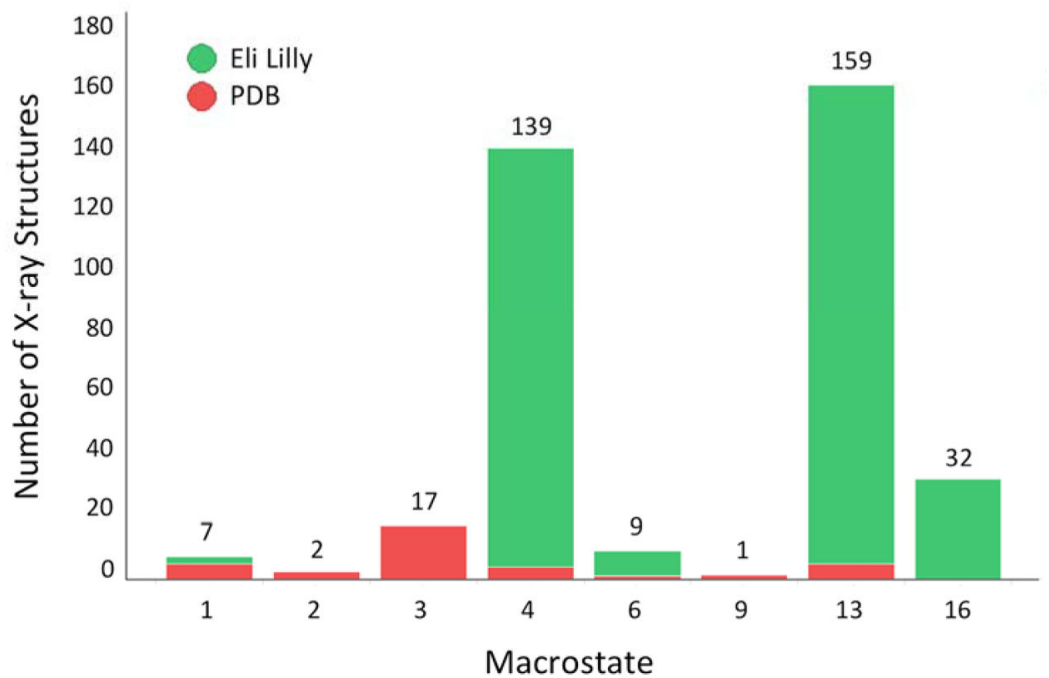

Figure 3.

Comparison of the macrostates with the available c-Abl X-ray structures which are from the public domain and from the Lilly internal structural database. X-ray structural propensity for each macrostate within structural databases. Red portion of bars show assignment of public structures to each macrostate. (one to one matching - each structure is assigned to closest macrostate), green bars show assignment of Lilly internal structures. 
a

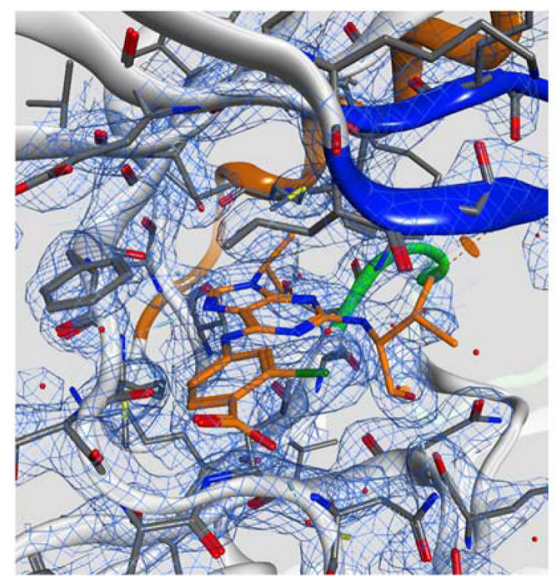

b

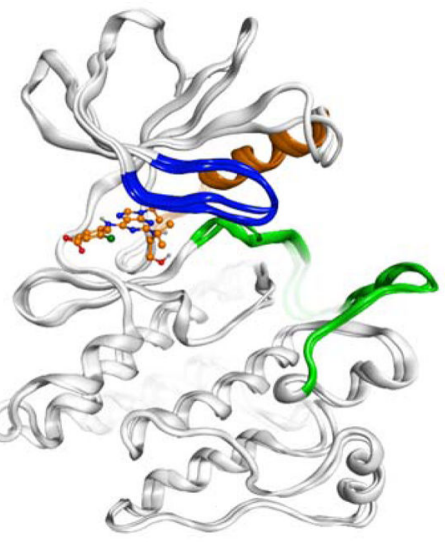

Figure 4.

Description of the X-ray crystallographic structure matching macrostate 16. 2Fo-Fc density was continuous and covered all WHI-P154 (2-chloro-4-\{[2-\{[(1r)-1-(hydroxymethyl)-2methylpropyl]amino -9-(1-methylethyl)-9h-purin-6-yl]amino \}benzoic acid) heavy atoms along with and the bulk of the main-chain and side chain residues within 10 angstroms of the ligand when contoured at 1.2 sigma. (a) Locl electron density in the neighborhood of the bound ligand. (b) Comparison of the structure of Abl1- WHI-P154 complex structure with macrostate16. The backbone RMSD between macrostate 16 and the X-ray structure is 0.73 $\AA$. The variable regions are depicted in colors (p-loop orange, A-loop cyan, aC-helix orange). The X-ray structure has been deposited in the PDB (6BL8). 
a

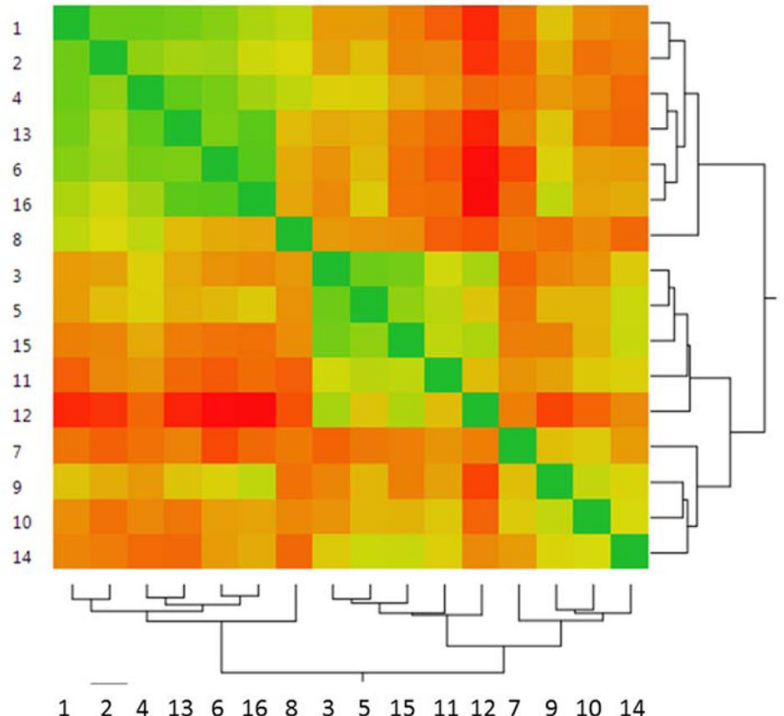

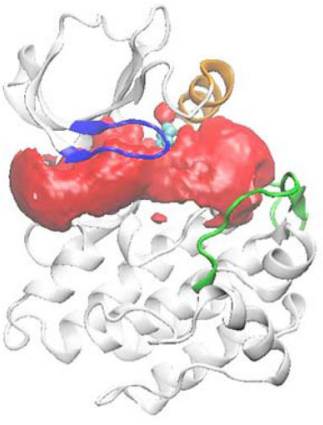

Macrostate 16

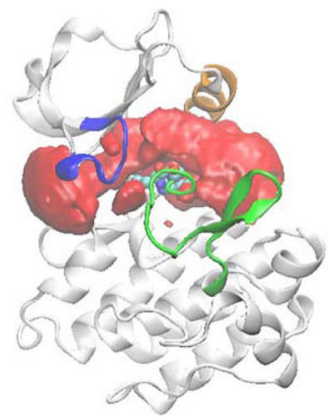

Macrostate 3

Figure 5.

Binding pocket properties of the macrostates. (a) Clustering of the binding pockets using the Tanimoto similarity metric (green $=1.0$, yellow $=0.75$, orange $=0.65$, red $=0.5$ ). (b) Depiction of the binding site of macrostate 16 (left) and macrostate 3 (right), which have a Tanimoto similarity of 0.65 . The same color scheme as utilized in Figure 4 was adopted for the variable region. 
a

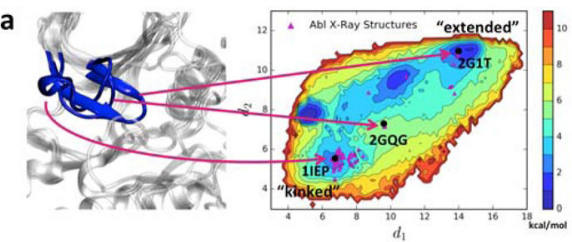

b

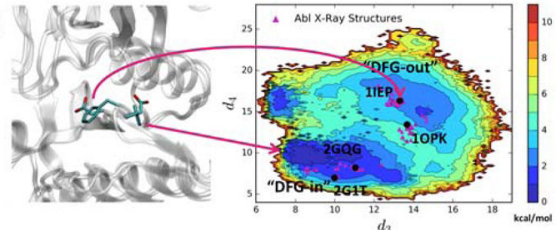

c

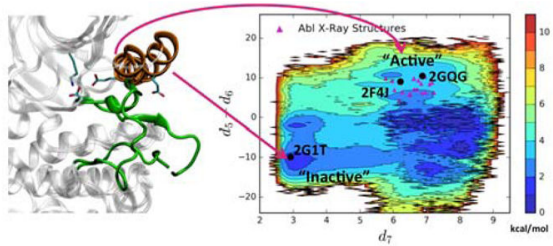

d

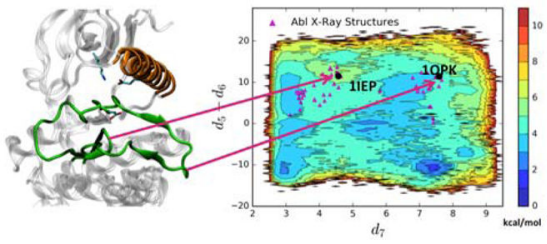

e

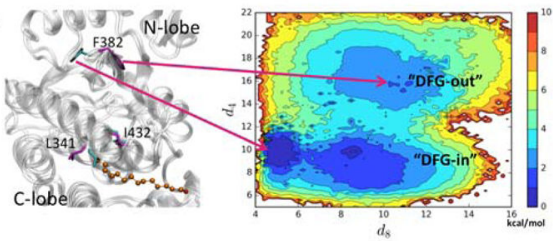

Figure 6.

Two-dimensional free energy landscapes underlying the dynamics of important structural motifs. (a) Conformations of the P-loop; (b) Conformation of the DFG (D381, F382, G383) motif; (c) Src/CDK-like activation (when the DFG motif is "in"); (d) Src/CDK-like transition (when the DFG motif is "out"); (e) Myristic acid is shown in the myristoylbinding site located in the C-lobe (the residues of the DFG motif are also shown). 


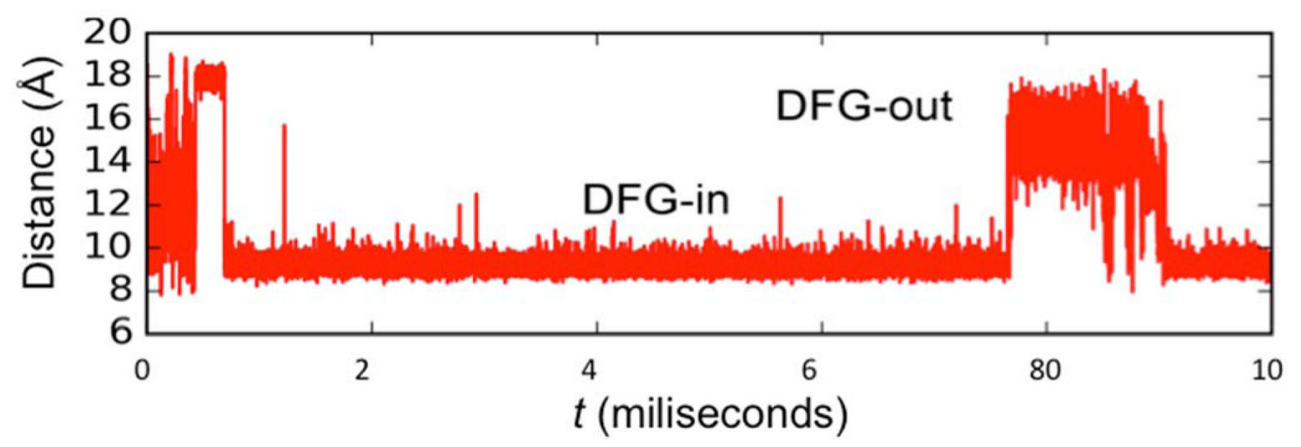

Figure 7.

Stochastic trajectory of DFG-flip in unphosphorylated apo-Abl kinase generated by the transition probability matrix of the MSM. The distance between C $\gamma$ of F382 and Ca of I293 was utilized to gauge the flip ( $d_{4}$ in Figure $6 \mathrm{~b}$ ). The lag-time used in the MSM stochastic trajectory is $40 \mathrm{~ns}$. 